\title{
Demographic-economic equilibria when the age at motherhood is endogenous ${ }^{1}$
}

\author{
Hippolyte d'Albis \\ Toulouse School of Economics (LERNA) \\ Emmanuelle Augeraud-Véron \\ University of La Rochelle (MIA) \\ Katheline Schubert \\ Paris School of Economics and University Paris 1 (CES) \\ First version: July 9, 2008 \\ This version: August 27, 2010
}

\footnotetext{
${ }^{1}$ We thank Noel Bonneuil, David de le Croix, the participants to the VID Viennese Vintage Workshop and the Overlapping Generations Days at Vielsalm, and two anonymous referees for their comments. The usual disclaimer applies.
} 


\begin{abstract}
In this article, we study the joint dynamics of the demography and the economy. We explore how economic conditions affect fertility choices, and in return how the population growth rate affects both financial and labor markets. Our main contribution is to consider a realistic demographic setup that allows characterizing the age at which individuals decide to give birth to their children. In such a framework, we aim at studying the existence of an equilibrium. We notably prove there exists a monetary steady state if the average age of consumers is greater than the average age of producers.
\end{abstract}




\section{Introduction}

In demographics, there is a long tradition of modeling population dynamics, dating back to the pioneer works of Alfred Lotka (1907, 1922). Stable population theory studies the dynamics of an age distribution once fertility and mortality patterns are held constant. Generalizations have then been proposed to take into account deterministic or stochastic changes in fertility and mortality. We propose to extend the traditional stable population model to endogenous fertility behaviors hinging on a trade-off between the utility derived from having children and the costs they induce. A key variable affecting both utility and costs is the age at which women become mothers. As pointed out by Gustafsson (2001), it is notably the age at first birth that is the main explanatory variable for the rapid decrease in fertility in developed countries. Using the data from the Human Fertility Database (2010), it is striking to see the negative correlation between the cohort's mean first maternal age and their total fertility rate. For instance, in the US, the mean age was slightly greater than 24 for cohorts born in 1918 as well as for those born in 1956, while it reached 22 and less for the cohorts, born between 1934 and 1940, that participated to the post-war baby-boom. In Figure 1 are represented the dynamics of the mean age at first birth and of the total fertility rates for cohorts born between 1918 and 1966.

We consider an overlapping generations model with continuous trading in which individuals work, consume and decide the age at motherhood. This choice affects labor participation and aggregate economic variables. We study the monetary equilibrium such that aggregate assets are positive. The main 


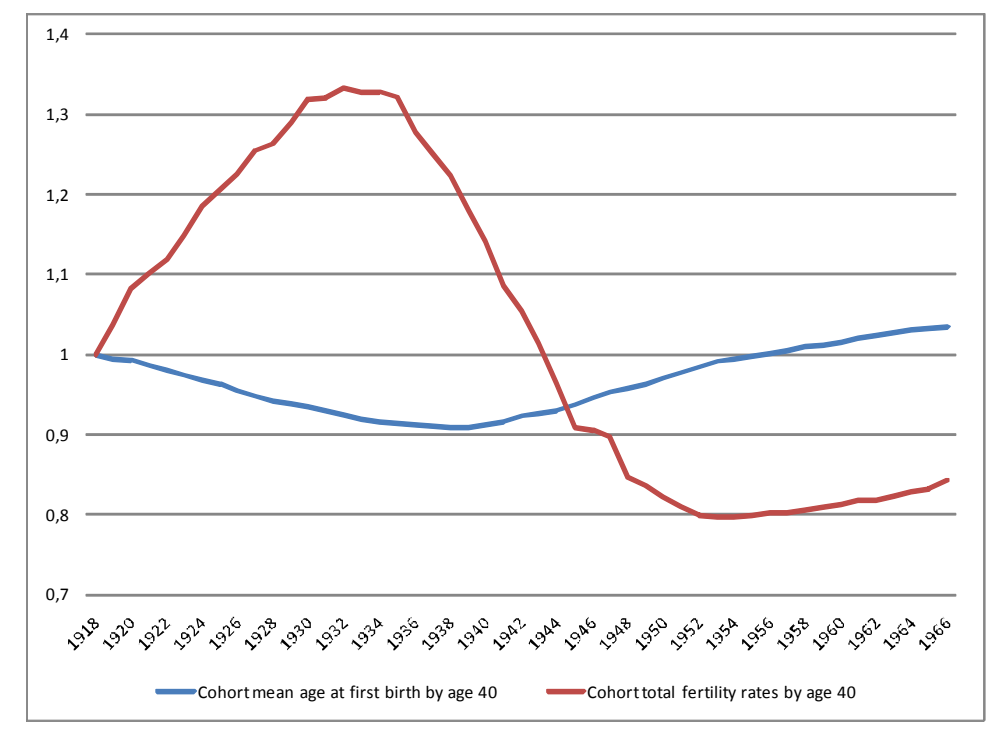

Figure 1: Evolution of cohorts's mean age at birth and fertility, USA. Source of data: Human Fertility Database (2010)

departure from Samuelson (1958) and the subsequent literature is that the population growth rate is endogenous.

The inter-temporal equilibrium is shown to be the solution of a nonlinear functional differential equation of mixed-type. The dynamics is indeed affected by discrete delays and advances. As in Boucekkine et al. (2002) and d'Albis and Augeraud-Véron (2007), delays are generated by the vintage structure of the population while advances rely on the expectations of the individuals. Moreover, with an endogenous age at motherhood, some of the delays and advances are state-dependent.

We characterize the steady states of our economy and focus on the monetary steady state. It is indeed well-known in the Samuelson (1958) setting that monetary steady states always appear as candidates for the equilibrium. In our framework with endogenous fertility, we show that it is not necessarily 
the case and that it depends on the marginal impact of the age at motherhood on human wealth. Moreover, we show that the condition initially exhibited by Arthur and McNicoll (1978) in a framework with exogenous population growth rate, still holds and is even necessary and sufficient for the existence of the monetary steady state. This condition says that the difference between the average age of consumers weighted by their consumptions and the average age of producers weighted by their earnings should be strictly positive. We finally show that the population growth rate obtained at the monetary equilibrium is always lower than the one that would be chosen by a social planner, upon existence of this latter solution.

Section 2 presents the model describing the individual life-cycle behavior and the aggregation of both the population and the economy. Section 3 studies the monetary equilibrium. Section 4 concludes.

\section{The model}

\subsection{Individual behaviour}

Individuals' lifetime is deterministic ${ }^{1}$, and of length 1 . Let $s$ be the birth date of the representative individual of generation $s$ and $s+\tau(s) \in[s, s+1]$ the date at which the individual born at $s$ gives birth to her $n(s)$ children. The number of children is supposed to be a decreasing function of the age at which the individual gives birth to her children. This assumption is captured

\footnotetext{
${ }^{1}$ We are aware that introducing some uncertain lifetime in accordance with Yaari (1965) would be not only more realistic (Boucekkine et al., 2004, d'Albis, 2007) but also might influence the results as argued by recent studies linking fertility to health conditions (Kalemli-Ozcan, 2003, Boucekkine et al., 2009). Specific difficulties arising with uncertain lifetime make nevertheless this issue beyond the scope of the paper.
} 
by function $f$, which satisfies:

$$
n(s)=f(\tau(s)), \quad f(.)>0, f^{\prime}(.)<0 .
$$

The individual born at $s$ derives utility from the flow of consumption $c(s, t)$ for $t \in[s, s+1]$ and from the lifetime spent with her children, i.e. $1-\tau(s)$. The intertemporal utility function is:

$$
U(s)=\int_{s}^{s+1} e^{-\rho(t-s)} u(c(s, t)) d t+\gamma v(1-\tau(s)),
$$

where $\rho \geq 0$ is the discount rate and where $\gamma>0$ is a parameter related to the preference for living with one's children relative to consumption. Assumptions about functions $u$ and $v$ are the following:

$$
u(c)=\mid \begin{aligned}
& \frac{c^{1-\frac{1}{\sigma}}-1}{1-\frac{1}{\sigma}} \text { if } \sigma \in(0,1) \cup(1,+\infty), \\
& \ln c \text { otherwise, }
\end{aligned}
$$

where $\sigma$ is the intertemporal elasticity of substitution of consumption, and

$$
v(.)>0, \quad v^{\prime}(.)>0, \quad \lim _{x \rightarrow 0} v^{\prime}(x)=+\infty, \quad v^{\prime \prime}(.)<0 .
$$

Each individual works along her entire lifetime, but her earnings are reduced for a given amount of time, denoted $\theta \in(0,1)$, when she becomes a mother. This can be explained by a decrease of the hours devoted to work during the early ages of the children. Let $\widetilde{w}(s, t, \tau(s))$ be the wage at time $t$ of the individual born at time $s$ and giving birth to her children at time $\tau(s)$ :

$$
\widetilde{w}(s, t, \tau(s))=\left\{\begin{array}{l}
\alpha(\tau(s)) w(t) \text { if } t-s \in[\tau(s), \min (\tau(s)+\theta, 1)] \\
w(t) \text { otherwise. }
\end{array}\right.
$$


Function $\alpha$ is a decreasing and convex function of the number of children, which is standard:

$$
\alpha(.) \in[0,1], \quad \alpha^{\prime}(.) \geq 0, \quad \lim _{x \rightarrow 0} \alpha^{\prime}(x)=+\infty, \quad \alpha^{\prime \prime}(.) \leq 0 .
$$

Individuals have access to a competitive asset market that yields $r(t)$, the interest rate. Let $a(s, t)$ denote the real wealth of an individual born at time $s$ as of time $t$. The instantaneous budget constraint is:

$$
\frac{\partial a(s, t)}{\partial t}=r(t) a(s, t)+\widetilde{w}(s, t, \tau(s))-c(s, t) .
$$

Individuals are born with no financial asset and cannot die indebted. Therefore, initial and terminal conditions write $a(s, s)=0$ and $a(s, s+1) \geq 0$. It is assumed that $a(s, t)$ and $c(s, t)$ are piecewise $\mathcal{C}^{1}\left(\mathbb{R}_{+}^{2}\right)$, and that $r(t)$ and $w(t)$ are continuous for all $t \in[s, s+1]$.

The individual born at $s$ seeks to maximize her intertemporal utility subject to her budget constraint and her initial and terminal conditions. The optimization problem reads:

$$
\begin{array}{l|l}
\max _{c(s, .), \tau(s)} \int_{s}^{s+1} e^{-\rho(t-s)} u(c(s, t)) d t+\gamma v(1-\tau(s)), & \begin{array}{l}
\frac{\partial a(s, t)}{\partial t}=r(t) a(s, t)+\widetilde{w}(s, t, \tau(s))-c(s, t), \\
\text { s.t. } \\
\widetilde{w}(s, t, \tau(s))=\left\{\begin{array}{l}
\alpha(\tau(s)) w(t) \text { if } t-s \in[\tau(s), \min (\tau(s)+\theta, 1)], \\
w(t) \text { otherwise, }
\end{array}\right. \\
c(s, t) \geq 0,0 \leq \tau(s) \leq 1, \\
a(s, s)=0, a(s, s+1) \geq 0 .
\end{array}
\end{array}
$$

It is convenient to define the human wealth at birth as follows:

$$
h(s, \tau(s))=\int_{s}^{s+1} R(z, s) \widetilde{w}(s, z, \tau(s)) d z,
$$


where $R(s, z)=\exp \left(\int_{s}^{z} r(u) d u\right)$ is the inverse of the discount factor.

We now present the solution of the individual's problem.

Lemma 1. The optimal consumption path, solution to problem $(\mathrm{P})$, satisfies:

$$
c(s, t)=\frac{R(s, t) h\left(s, \tau^{*}(s)\right)}{\int_{s}^{s+1} R(z, t)^{1-\sigma} e^{-\sigma \rho(z-t)} d z},
$$

where $\tau^{*}(s) \in(0,1)$ is the solution of:

$$
c(s, s)^{-\frac{1}{\sigma}} \frac{\partial h(s, \tau(s))}{\partial \tau(s)}-\gamma v^{\prime}(1-\tau(s))=0 .
$$

Moreover, $\tau^{*}(s)$ is unique if:

$$
\frac{\partial^{2} h(s, \tau(s))}{\partial \tau^{2}(s)} \leq 0 .
$$

Proof. The method is similar to the one used by d'Albis and Augeraud-Véron (2008) for endogenous retirement. The problem is solved in two steps: first, for a given $\tau(s)$, the optimal path of consumption is computed; then, the optimal age at which the agent gives birth to her children is derived.

Step 1. The optimal path of consumption for any $\tau(s)$ (or equivalently for a given path $\widetilde{w}(s, z, \tau(s)))$ is derived from the Euler's equation, and satisfies at age $t-s$ :

$$
c(s, t)=c(s, s) R(s, t)^{\sigma} e^{-\sigma \rho(t-s)},
$$

while the intertemporal budget constraint is obtained by integrating forward (7) and using the following optimal equality: $a(s, s+1)=0$, such that:

$$
\int_{s}^{s+1} R(z, s) c(s, z) d z=h(s, \tau(s)) .
$$

By replacing (12) in (13), we obtain the optimal initial consumption:

$$
c(s, s)=\frac{h(s, \tau(s))}{\int_{s}^{s+1} R(z, s)^{1-\sigma} e^{-\sigma \rho(z-s)} d z} .
$$


With (12) and (14), we obtain (9).

Step 2. The optimal age $\tau(s)$ is obtained by replacing (9) in (2) and solving the following problem:

$$
\begin{aligned}
& \max _{\tau(s)} \int_{s}^{s+1} e^{-\rho(t-s)} \frac{c(s, t)^{1-\frac{1}{\sigma}}}{1-\frac{1}{\sigma}} d t+\gamma v(1-\tau(s)), \\
& \begin{array}{l}
c(s, t)=c(s, s) R(s, t)^{\sigma} e^{-\sigma \rho(t-s)}, \\
c(s, s)=\frac{h(s, \tau(s))}{\int_{s}^{s+1} R(z, s)^{1-\sigma} e^{-\sigma \rho(z-s)} d z}, \\
\widetilde{w}(s, z, \tau(s))=\left\{\begin{array}{l}
\alpha(\tau(s)) w(z) \text { if } z-s \in[\tau(s), \min (\tau(s)+\theta, 1)], \\
w(z) \text { otherwise, } \\
0 \leq \tau(s) \leq 1 .
\end{array}\right.
\end{array}
\end{aligned}
$$

Let $\lambda_{1} \geq 0$ and $\lambda_{2} \geq 0$ be the two Kuhn and Tucker multipliers associated to the inequality constraints on $\tau(s)$. The first order condition is:

$$
c(s, s)^{-\frac{1}{\sigma}} \frac{\partial c(s, s)}{\partial \tau(s)} \int_{s}^{s+1} R(s, t)^{\sigma-1} e^{-\sigma \rho(t-s)} d t-\gamma v^{\prime}(1-\tau(s))+\lambda_{1}-\lambda_{2}=0
$$

i.e.

$$
c(s, s)^{-\frac{1}{\sigma}} \frac{\partial h(s, \tau(s))}{\partial \tau(s)}-\gamma v^{\prime}(1-\tau(s))+\lambda_{1}-\lambda_{2}=0
$$

and the two complementarity slackness conditions are:

$$
\begin{aligned}
& \lambda_{1} \geq 0, \tau(s) \geq 0, \lambda_{1} \tau(s)=0 \\
& \lambda_{2} \geq 0,1-\tau(s) \geq 0, \lambda_{2}(1-\tau(s))=0 .
\end{aligned}
$$

Let us first establish the existence condition for an interior solution.

We first note that $\lambda_{2}>0$ is never optimal. Indeed, if $\lambda_{2}>0, \tau(s)=1$ and $\lambda_{1}=0$, and since $\lim _{\tau(s) \rightarrow 1} v^{\prime}(1-\tau(s))=+\infty$ we obtain a contradiction. 
We then note than $\lambda_{1}>0$ cannot be optimal either. Indeed, if $\lambda_{1}>0$, $\tau(s)=0$ and $\lambda_{2}=0$, and condition (16) reads

$$
\lambda_{1}=\gamma v^{\prime}(1)-\lim _{\tau(s) \rightarrow 0}\left[c(s, s)^{-\frac{1}{\sigma}} \frac{\partial h(s, \tau(s))}{\partial \tau(s)}\right] .
$$

According to (8) and (5), we have:

$$
\begin{aligned}
h(s, \tau(s))= & \int_{s}^{s+\tau(s)} R(z, s) w(z) d z+\alpha(\tau(s)) \int_{s+\tau(s)}^{s+\min ((\tau(s)+\theta), 1)} R(z, s) w(z) d z \\
& +\int_{s+\min ((\tau(s)+\theta), 1)}^{s+1} R(z, s) w(z) d z .
\end{aligned}
$$

Then

$$
\begin{aligned}
\frac{\partial h(s, \tau(s))}{\partial \tau(s)}= & (1-\alpha(\tau(s)))[R(s+\tau(s), s) w(s+\tau(s)) \\
& \left.-\frac{1-\min ((\tau(s)+\theta), 1)}{1-(\tau(s)+\theta)} R(s+\tau(s)+\theta, s) w(s+\tau(s)+\theta)\right] \\
& +\alpha^{\prime}(\tau(s)) \int_{s+\tau(s)}^{s+\min ((\tau(s)+\theta), 1)} R(z, s) w(z) d z .
\end{aligned}
$$

As $\lim _{x \rightarrow 0} \alpha^{\prime}(x)=+\infty$, we obtain that $\lim _{\tau(s) \rightarrow 0} \partial h(s, \tau(s)) / \partial \tau(s)=+\infty$ and $\lambda_{1} \rightarrow-\infty$, a contradiction.

Concerning the uniqueness of the interior solution, we simply need to ensure that the left-hand side of (10) decreases with $\tau(s)$. Let $G(\tau(s))$ be this lefthand side. We have

$$
\begin{aligned}
G^{\prime}(\tau(s))= & -\frac{1}{\sigma} c(s, s)^{-\frac{1}{\sigma}-1} \frac{\partial c(s, s)}{\partial \tau(s)} \frac{\partial h(s, \tau(s))}{\partial \tau(s)} \\
& +c(s, s)^{-\frac{1}{\sigma}} \frac{\partial^{2} h(s, \tau(s))}{\partial \tau(s)^{2}}+\gamma v^{\prime \prime}(1-\tau(s)),
\end{aligned}
$$

with $\frac{\partial c(s, s)}{\partial \tau(s)}$ of the same sign as $\frac{\partial h(s, \tau(s))}{\partial \tau(s)}$ (see equation $\left.(14)\right)$ and $v^{\prime \prime}()<$.0 by assumption. Then $G^{\prime}(\tau(s))$ is strictly negative if condition (11) is satisfied. 
The problem of the individual is to find the optimal trade-off between consuming and spending time with her children. A key variable is thus the influence of the age at motherhood on the human wealth. Due to parameter $\theta$, which captures the time spent raising children, this influence is not straightforward. Indeed, for early motherhood, or equivalently for low $\theta$, there could exist wage and interest rate profiles such that $\partial h(s, \tau(s)) / \partial \tau(s)$ is negative (see equation (19)). In that case, the optimal solution would be to choose $\tau(s)=0$ because it allows the individual to both consume more and spend more time with her children. Assumption $\lim _{x \rightarrow 0} \alpha^{\prime}(x)=+\infty$ excludes this corner solution. Finally, the concavity of human wealth with respect to the age at motherhood is sufficient for excluding multiple local optima to problem (P) as it implies a unique solution to equation (10).

It will be useful to compute the optimal path of asset during the lifespan. Since the intertemporal budget constraint is:

$$
a(s, t)=\int_{t}^{s+1} R(z, t)[c(s, z)-\widetilde{w}(s, z, \tau(s))] d z,
$$

one obtains:

$$
\begin{aligned}
a(s, t)= & R(s, t) h\left(s, \tau^{*}(s)\right) \int_{t}^{s+1}\left[\int_{s}^{s+1} R(u, z)^{1-\sigma} e^{-\sigma \rho(u-z)} d u\right]^{-1} d z \\
& -\int_{t}^{s+1} R(z, t) \widetilde{w}(s, z, \tau(s)) d z
\end{aligned}
$$

\subsection{Aggregate variables}

\subsubsection{The demography}

The demographic structure is in overlapping generations.

Let $N(t)$ be the size of the cohort born at time $t$. Let $T(t), \beta(t)$ and $\nu(t)$ be respectively the size of the population, the crude birth rate and the 
population growth rate such that:

$$
\begin{aligned}
T(t) & =\int_{t-1}^{t} N(s) d s, \\
\beta(t) & =\frac{N(t)}{T(t)}, \\
\nu(t) & =\frac{N(t)-N(t-1)}{T(t)} .
\end{aligned}
$$

The relationship between the birth and the growth rates can be written as:

$$
\beta(t)-\beta(t-1) e^{-\int_{t-1}^{t} \nu(u) d u}=\nu(t),
$$

which yields to a delay differential equation when differentiated once more. The cohort born at $t$ gives birth to a new cohort at date $t+\tau(t)$. The size of the latter satisfies $N(t+\tau(t))=f(\tau(t)) N(t)$, or equivalently

$$
\beta(t+\tau(t)) e^{\int_{t}^{t+\tau(t)} \nu(u) d u}=f(\tau(t)) \beta(t)
$$

which is a differential equation with endogenous advances. Let us remark that this framework allows for the existence of more than two generations at the same time: for instance if $\tau(t)<1 / 2$, an individual will survive her grand daughter.

\subsubsection{The economy}

It is supposed that the production is equal to the efficient labor force ${ }^{2}$. The productivity of an individual without children is normalized to 1 and bearing a child has an opportunity cost which reduces the time devoted to production. Moreover, there is no redistribution. In this framework, wages are constant: $w(t)=1$, for all $t$.

\footnotetext{
${ }^{2}$ Such economies are sometime referred to as "fruit tree" or "yeoman farmer" economies.
} 
We assume that the cohort which decides to have children at $t$ is the youngest one among those who have children at $t$. This so-called "last in, last out" assumption is strong but it is satisfied in the neighborhood of the steady state $^{3}$.

With these assumptions, let us define total production at date $t$ :

$$
Y(t)=\int_{t-1}^{\max (\eta, t-1)} N(s) d s+\int_{\max (\eta, t-1)}^{x} \alpha(\tau(s)) N(s) d s+\int_{x}^{t} N(s) d s
$$

where $x$ is the birth date of the cohort who decided to have their children at date $t$ and $\eta$ is the birth date of the cohort who had their children at date $t-\theta$, and whose productivity is not reduced anymore. The first integral in equation (24) is, upon existence, the production of the eldest generations who do not take care anymore of their children, the second integral is the production of the parents generations who are still taking care of their children, while the third integral is the production of the youngest cohorts who do not have their children yet (they are born after $x$ ). Dates $x$ and $\eta$ respectively satisfy:

$$
x+\tau(x)=t \text { and } \eta+\tau(\eta)+\theta=t .
$$

We remark that production can equivalently be written as follows:

$$
\begin{aligned}
Y(x+\tau(x))= & \int_{x+\tau(x)-1}^{\max (\eta, x+\tau(x)-1)} N(s) d s+\int_{\max (\eta, x+\tau(x)-1)}^{x} \alpha(\tau(s)) N(s) d s \\
& +\int_{x}^{x+\tau(x)} N(s) d s
\end{aligned}
$$

which is an integral equation with endogenous lags and leads.

\footnotetext{
${ }^{3}$ Indeed, by definition, the lag $\tau(t)$ is constant at the steady-state.
} 


\section{Monetary equilibrium}

In this section, we define the intertemporal equilibria, characterize the steady states, and focus on the monetary one. Monetary steady states are key in the OLG literature, where they are known to be candidates for the equilibrium. Studying them is thus the easiest way to prove the existence of an equilibrium. Moreover, monetary steady states are known to be optimal in the standard framework with exogenous fertility. We show that it is not the case when the age at motherhood is endogenous.

\subsection{The intertemporal equilibrium}

Denote by $y(t), c(t)$ and $a(t)$ the per capita aggregate consumption, production and assets, respectively, with for instance $c(t)=\frac{\int_{t-1}^{t} N(s) c(s, t) d s}{T(t)}=\int_{t-1}^{t} \beta(s) \frac{T(s)}{T(t)} c(s, t) d s=\int_{t-1}^{t} \beta(s) e^{-\int_{s}^{t} \nu(u) d u} c(s, t) d s$.

Definition 1. An intertemporal equilibrium is a path such that individuals behave optimally and the goods market clears: $c(t)=y(t)$. The intertemporal equilibrium is classical if $a(t)=0$ and monetary if $a(t)>0$.

The equilibrium is the solution of a system in $(r(t), \tau(t), \beta(t), \nu(t))$ composed by two "economic" equations and two demographic equations. The economic part is featured by the optimal age at motherhood, which using (10), (14) and the definition of $h(s, \tau(s))$ given by (8), can be written as:

$$
\left(\frac{h(s, \tau(s))}{\int_{s}^{s+1} R(z, s)^{1-\sigma} e^{-\sigma \rho(z-s)} d z}\right)^{-\frac{1}{\sigma}} \frac{\partial h(s, \tau(s))}{\partial \tau(s)}-\gamma v^{\prime}(1-\tau(t))=0,
$$


and the equilibrium on the goods market:

$$
\begin{gathered}
\int_{t-1}^{t} \beta(s) e^{-\int_{s}^{t} \nu(u) d u} \frac{R(s, t) h(s, \tau(s))}{\int_{s}^{s+1} R(z, t)^{1-\sigma} e^{-\sigma \rho(z-t)} d z} d s= \\
\int_{t-1}^{\max (\eta, t-1)} \beta(s) e^{-\int_{s}^{t} \nu(u) d u} d s+\int_{\max (\eta, t-1)}^{x} \alpha(\tau(s)) \beta(s) e^{-\int_{s}^{t} \nu(u) d u} d s+\int_{x}^{t} \beta(s) e^{-\int_{s}^{t} \nu(u) d u} d s,
\end{gathered}
$$

where $x+\tau(x)=t$ and $\eta+\tau(\eta)+\theta=t$. Moreover, the two demographic equations are given by (22) and (23). Finally, using (21), the aggregate asset per capita is given by:

$$
\begin{aligned}
a(t)= & \int_{t-1}^{t} \beta(s) e^{-\int_{s}^{t} \nu(u) d u} a(s, t) d s \\
= & \int_{t-1}^{t} \beta(s) e^{-\int_{s}^{t} \nu(u) d u}\left[R(s, t) h(s, \tau(s)) \int_{t}^{s+1}\left[\int_{s}^{s+1} R(u, z)^{1-\sigma} e^{-\sigma \rho(u-z)} d u\right]^{-1} d z\right] d s \\
& -\int_{t-1}^{t} \beta(s) e^{-\int_{s}^{t} \nu(u) d u}\left[\int_{t}^{s+1} R(z, t) \widetilde{w}(s, z, \tau(s)) d z\right] d s,
\end{aligned}
$$

which turns to be:

$$
\begin{aligned}
a(t)= & \int_{t-1}^{t} \beta(s) e^{-\int_{s}^{t} \nu(u) d u}\left[R(s, t) h\left(s, \tau^{*}(s)\right) \int_{t}^{s+1}\left[\int_{s}^{s+1} R(u, z)^{1-\sigma} e^{-\sigma \rho(u-z)} d u\right]^{-1} d z\right] d s \\
& -\int_{t-1}^{\max (\eta, t-1)} \beta(s) e^{-\int_{s}^{t} \nu(u) d u}\left[\int_{t}^{s+1} R(z, t) d z\right] d s \\
& -\int_{\max (\eta, t-1)}^{x} \beta(s) e^{-\int_{s}^{t} \nu(u) d u}\left[\alpha(\tau(s)) \int_{t}^{s+\min (\tau(s)+\theta, 1)} R(z, t) d z\right. \\
& \left.+\int_{s+\min (\tau(s)+\theta, 1)}^{s+1} R(z, t) d z\right] d s \\
& -\int_{x}^{t} \beta(s) e^{-\int_{s}^{t} \nu(u) d u}\left[\int_{t}^{s+\tau(s)} R(z, t) d z\right. \\
& \left.+\alpha(\tau(s)) \int_{s+\tau(s)}^{s+\min (\tau(s)+\theta, 1)} R(z, t) d z+\int_{s+\min (\tau(s)+\theta, 1)}^{s+1} \quad R(z, t) d z\right] d s
\end{aligned}
$$




\subsection{Characterization of steady states}

Definition 2. A steady state is an intertemporal equilibrium such that $(r(t), \tau(t), \beta(t), \nu(t))$ is constant.

Property 1. A steady state is a solution of the following system:

$$
\begin{gathered}
\left(\frac{h(\tau)}{\int_{0}^{1} e^{-[(1-\sigma) r+\sigma \rho] z} d z}\right)^{-\frac{1}{\sigma}}\left\{(1-\alpha(\tau))\left[e^{-r \tau}-\frac{1-\min (\tau+\theta, 1)}{1-(\tau+\theta)} e^{-r(\tau+\theta)}\right]\right. \\
\left.+\alpha^{\prime}(\tau) \int_{\tau}^{\min ((\tau+\theta), 1)} e^{-r z} d z\right\}-\gamma v^{\prime}(1-\tau)=0, \\
f(\tau)-e^{\nu \tau}=0 \\
h(\tau) \int_{-1}^{0} \frac{e^{(\nu-r) s}}{\int_{s}^{s+1} e^{-[(1-\sigma) r+\sigma \rho] z} d z} d s \\
-\left[\int_{-1}^{-\min (\tau+\theta, 1)} e^{\nu s} d s+\alpha(\tau) \int_{-\min (\tau+\theta, 1)}^{-\tau} e^{\nu s} d s+\int_{-\tau}^{0} e^{\nu s} d s\right]=0
\end{gathered}
$$

with

$$
h(\tau)=\int_{0}^{\tau} e^{-r z} d z+\alpha(\tau) \int_{\tau}^{\min (\tau+\theta, 1)} e^{-r z} d z+\int_{\min (\tau+\theta, 1)}^{1} e^{-r z} d z .
$$

Proof. At steady state, $r$ is constant, which implies that $R(t, s)=e^{-r(t-s)}$. Equation (27) of the dynamic system, characterizing the individual choice of $\tau$, hence writes:

$$
\left(\frac{h(s, \tau)}{\int_{s}^{s+1} e^{-[(1-\sigma) r+\sigma \rho](z-s)} d z}\right)^{-\frac{1}{\sigma}} \frac{\partial h(s, \tau)}{\partial \tau}-\gamma v^{\prime}(1-\tau)=0 .
$$

Using (8), we obtain (30). Equations (22) and (23) write respectively: $\beta=$ $\nu /\left(1-e^{-\nu}\right)$ and $f(\tau)=e^{\nu \tau}$. The latter is equation (31), while the former gives the value of the birth rate $\beta$ at steady state, once $\nu$ has been calculated 
through system (30)-(32). Similarly, equation (28), featuring the equilibrium condition, can be rewritten to obtain (32).

We finally note that aggregate asset per capita, given by equation (29), satisfies:

$$
\begin{aligned}
\frac{a}{\beta}= & h(\tau) \int_{-1}^{0} e^{(\nu-r) s}\left[\int_{0}^{s+1}\left[\int_{s}^{s+1} e^{-[(1-\sigma) r+\sigma \rho](u-z)} d u\right]^{-1} d z\right] d s \\
& -\int_{-1}^{-\min (\tau+\theta, 1)} e^{\nu s}\left[\int_{0}^{s+1} e^{-r z} d z\right] d s \\
& -\int_{-\min (\tau+\theta, 1)}^{-\tau} e^{\nu s}\left[\alpha(\tau) \int_{0}^{s+\min (\tau+\theta, 1)} e^{-r z} d z+\int_{s+\min (\tau+\theta, 1)}^{s+1} e^{-r z} d z\right] d s \\
& -\int_{-\tau}^{0} e^{\nu s}\left[\int_{0}^{s+\tau} e^{-r z} d z+\alpha(\tau) \int_{s+\tau}^{s+\min (\tau+\theta, 1)} e^{-r z} d z+\int_{s+\min (\tau+\theta, 1)}^{s+1} e^{-r z} d z\right] d s .
\end{aligned}
$$

\subsection{Existence of a monetary steady state}

Lemma 2. There exists a candidate for the monetary steady state if $\sigma \geq 1$ or if $\sigma<1$ and $\lim _{\tau \rightarrow 0} \tau^{1+\sigma} \alpha^{\prime}(\tau)^{\sigma}=+\infty$. It corresponds to a positive demographic growth rate if and only if $\gamma>\bar{\gamma}$, where $\bar{\gamma}>0$.

Proof. For the first part of the lemma, we look for particular solutions of system (30)-(32), such that: $r=\nu$. We first check that equation (32) is always satisfied when $r=\nu$. The system then reduces to equation (30), written for $r=\nu$, and (31).

Let us first study the case of the particular solution $\nu=0$. It is obtained for the particular age, denoted $\bar{\tau}$, which satisfies: $f(\bar{\tau})=1$. Define moreover the parameter $\xi=\min (\theta, 1-\bar{\tau})$. Then, $\nu=0$ is a solution if and only if:

$$
\left(\frac{1-(1-\alpha(\bar{\tau})) \xi}{\int_{0}^{1} e^{-\sigma \rho z} d z}\right)^{-\frac{1}{\sigma}}\left[(1-\alpha(\bar{\tau})) \frac{\xi-\theta}{(1-\bar{\tau})-\theta}+\alpha^{\prime}(\bar{\tau}) \xi\right]-\gamma v^{\prime}(1-\bar{\tau})=0
$$


which is, of course, not generic. We denote by $\bar{\gamma}$ the unique value of the parameter of preference for living with one's children ensuring that equation (36) is satisfied.

Let us then define function $F$ as:

$$
\begin{aligned}
F(\tau)= & \frac{1-e^{-\left((1-\sigma) \frac{\ln f(\tau)}{\tau}+\sigma \rho\right)}}{(1-\sigma) \frac{\ln f(\tau)}{\tau}+\sigma \rho} f(\tau)^{1-\sigma} \ln f(\tau)\left[v^{\prime}(1-\tau)\right]^{-\sigma} \\
& \times\left[(1-\alpha(\tau))\left[1-\frac{1-\min (\tau+\theta, 1)}{1-(\tau+\theta)} f(\tau)^{-\frac{\theta}{\tau}}\right]+\tau \alpha^{\prime}(\tau) \frac{1-f(\tau)^{1-\frac{\min (\tau+\theta, 1)}{\tau}}}{\ln f(\tau)}\right]^{\sigma} \\
& -\tau \gamma^{\sigma}\left[f(\tau)-f(\tau)^{1-\frac{1}{\tau}}+(1-\alpha(\tau))\left(f(\tau)^{1-\frac{\min (\tau+\theta, 1)}{\tau}}-1\right)\right]
\end{aligned}
$$

where $F$ is continuous. Equations (30) with $r=\nu$ and (31) are equivalent to $F(\tau)=0$, with $\tau \neq 0$ and $f(\tau) \neq 1$. Let us study function $F(\tau)$. Since $F(\bar{\tau})=0$ and $F(1)=\gamma^{\sigma}(1-f(1))>0$, a sufficient condition for the existence of a $\tau^{*}$, which belongs to $(0, \bar{\tau}) \cup(\bar{\tau}, 1)$ and satisfies $F\left(\tau^{*}\right)=0$, is $F(0)>0$. We have, as $f(0)>1$ :

$$
\begin{aligned}
F(0)= & \lim _{\tau \rightarrow 0}\left(\frac{1-e^{-\left[(1-\sigma) \frac{\ln f(0)}{\tau}+\sigma \rho\right]}}{(1-\sigma) \frac{\ln f(0)}{\tau}+\sigma \rho}\right) f(0)^{1-\sigma} \ln f(0)\left[v^{\prime}(1)\right]^{-\sigma} \\
& \times\left[(1-\alpha(0))+\lim _{\tau \rightarrow 0}\left(\tau \alpha^{\prime}(\tau)\right) \frac{1}{\ln f(0)}\right]^{\sigma} .
\end{aligned}
$$

Since

$$
\lim _{\tau \rightarrow 0} \frac{1-e^{-\left[(1-\sigma) \frac{\ln f(0)}{\tau}+\sigma \rho\right]}}{(1-\sigma) \frac{\ln f(0)}{\tau}+\sigma \rho}=\left\{\begin{array}{l}
0, \text { if } \sigma<1, \\
\frac{1-e^{-\rho}}{\rho}, \text { if } \sigma=1, \\
+\infty, \text { if } \sigma>1,
\end{array}\right.
$$

we obtain that $F(0)>0$ if $\sigma \geq 1$. The case $\sigma<1$ is more complicated because we have supposed that $\lim _{\tau \rightarrow 0} \alpha^{\prime}(\tau)=+\infty$. Hence, $F(0)$ is either 0 or $+\infty$. The condition $\lim _{\tau \rightarrow 0} \tau^{1+\sigma} \alpha^{\prime}(\tau)^{\sigma}=+\infty$ ensures that $F(0)=+\infty$. For the second part of the lemma, we use the fact that if $F(0)>0, \tau^{*} \leq \bar{\tau}$ (and, consequently, $\nu \geq 0$ ) if and only if $F^{\prime}(\bar{\tau}) \geq 0$. We then compute the 
first derivative of (37) at point $\bar{\tau}$ such that ${ }^{4}$ :

$$
\begin{aligned}
F^{\prime}(\bar{\tau})= & \frac{1-e^{-\sigma \rho}}{\sigma \rho}\left[(1-\alpha(\bar{\tau})) \frac{\xi-\theta}{(1-\bar{\tau})-\theta}+\alpha^{\prime}(\bar{\tau}) \xi\right]^{\sigma}\left[v^{\prime}(1-\bar{\tau})\right]^{-\sigma} f^{\prime}(\bar{\tau}) \\
& -\gamma^{\sigma}(1-(1-\alpha(\bar{\tau})) \xi) f^{\prime}(\bar{\tau})
\end{aligned}
$$

We thus conclude that

$$
F^{\prime}(\bar{\tau}) \geq 0 \Leftrightarrow \gamma \geq \bar{\gamma}
$$

$\bar{\gamma}$ defined by equation (36) is the threshold of the parameter of preference for living with one's children such that $F^{\prime}(\bar{\tau}) \geq 0$ if and only if $\gamma \geq \bar{\gamma}$.

Due to the endogeneity of the demographic growth rate, the existence of a monetary steady state is not as straightforward as in the standard case. The problem appears when the elasticity of intertemporal substitution is lower than one. This lowers individual savings and thus increases the interest rate, which is at the equilibrium the demographic growth rate. This is only possible if the age at motherhood is reduced. To reach a sufficiently large demographic growth rate, the age should be zero, which is forbidden, as shown in Lemma 1 , by the assumption $\lim _{\tau \rightarrow 0} \alpha^{\prime}(\tau)=+\infty$. To avoid the incompatibility that may arise with a low savings behavior and an interior solution for the age at motherhood we impose a condition that simply says that the convergence of $\alpha^{\prime}(\tau)$ to $+\infty$ is sufficiently fast.

The second part of the lemma is more intuitive. The preference for spending time with children must be large enough to yield a positive demographic growth. Moreover, when the time spent raising one's children is not too large $(\theta<1-\bar{\tau})$, the threshold parameter above which population growth is pos-

\footnotetext{
${ }^{4}$ The proof, involving simple but very tedious calculations, is available on demand.
} 
itive increases with $\theta$. The threshold is of course independent of $\theta$ when $\theta$ is so large that the mother cannot die before having raised her children.

For the next result, it is useful to define $\bar{\alpha}_{x}$, the average age calculated cross-sectionally such that:

$$
\bar{\alpha}_{x} \equiv \frac{\int_{t-1}^{t}(t-s) x(s, t) N(s) d s}{\int_{0}^{1} x(s, t) N(s) d s},
$$

where $x(s, t)$ is a relevant characteristic. In the case $x(s, t)=1, \bar{\alpha}_{1}$ is the standard definition of the average age of the population. If $x(s, t)=c(s, t)$, $\bar{\alpha}_{c}$ is the average age of consumers weighted by their consumptions and equivalently, if $x(s, t)=y(s, t), \bar{\alpha}_{y}$ is the average age of producers weighted by their earnings. We obtain:

$$
\begin{aligned}
\bar{\alpha}_{1} & =\frac{\int_{t-1}^{t}(t-s) e^{-\nu(t-s)} d s}{\int_{0}^{1} e^{-\nu(t-s)} d s}=\frac{1}{\nu}-\frac{1}{e^{\nu}-1} \\
\bar{\alpha}_{c} & =\frac{1}{(1-\sigma) \nu+\sigma \rho}-\frac{1}{e^{[(1-\sigma) \nu+\sigma \rho]}-1}, \\
\bar{\alpha}_{y} & =\frac{1}{\nu}-\frac{e^{-\nu}+(1-\alpha(\tau)) e^{-\nu \tau}\left(\tau-(\tau+\min (\theta, 1-\tau)) e^{-\nu \min (\theta, 1-\tau)}\right.}{1-e^{-\nu}-(1-\alpha(\tau)) e^{-\nu \tau}\left(1-e^{-\nu \min (\theta, 1-\tau)}\right)}
\end{aligned}
$$

Notice that when there is no population growth, the average age of the population is $1 / 2$ while the average age of consumers always belongs to $(0,1 / 2)$. When impatience is high, agents wish to consume early in their lifespan, and the average age of consumers is all the more lower than the average age of the population. More generally, the average age of consumers belongs to $(0,1)$ and is a decreasing function of $(1-\sigma) \nu+\sigma \rho^{5}$. Thus it increases with $\nu$ if $\sigma>1$ and decreases with $\nu$ if $\sigma<1$. This can be explained by the

\footnotetext{
${ }^{5}$ Indeed, one has $\bar{\alpha}_{c}=g(x)=\frac{\left(e^{x}-1\right)-x}{x\left(e^{x}-1\right)}$ where $x=(1-\sigma) \nu+\sigma \rho$. Function $g$ is decreasing, with $\lim _{x \rightarrow-\infty} g(x)=1, g(0)=1 / 2$ and $\lim _{x \rightarrow+\infty} g(x)=0$.
} 
fact that $\nu$ is both the growth rate of the population and the equilibrium interest rate. Consequently, if the intertemporal elasticity of substitution is sufficiently large, the increase of consumption over the life cycle exceeds the change in the demographic structure and finally the average age of consumers increases with $\nu$.

Proposition 1. The candidate corresponds to a valid monetary steady state if and only if $\bar{\alpha}_{c}>\bar{\alpha}_{y}$.

Proof. The candidate corresponds to a valid monetary steady state if and only if the aggregate asset per capita is positive. When $\nu=r$, equation (35) rewrites as follows:

$$
\begin{aligned}
\left(1-e^{-\nu}\right) a= & \nu h(\tau)\left[\frac{1}{(1-\sigma) \nu+\sigma \rho}-\frac{1}{e^{[(1-\sigma) \nu+\sigma \rho]}-1}\right] \\
& -h(\tau)+e^{-\nu}+(1-\alpha(\tau)) e^{-\nu \tau} \\
& \times\left[\left(1-e^{-\nu \min (\theta, 1-\tau)}\right) \tau-e^{-\nu \min (\theta, 1-\tau)} \min (\theta, 1-\tau)\right]
\end{aligned}
$$

with

$$
h(\tau)=\frac{1-e^{-\nu}-(1-\alpha(\tau)) e^{-\nu \tau}\left(1-e^{-\nu \min (\theta, 1-\tau)}\right)}{\nu}
$$

Using (43) and (44), this rewrites

$$
a=\left(\bar{\alpha}_{c}-\bar{\alpha}_{y}\right) \frac{\nu}{1-e^{-\nu}} h(\tau)
$$

Among stationary solutions, the Golden Rule path, such that the interest rate equals the demographic growth rate, has been extensively studied. Since the work of Samuelson (1958), it is customary to name this solution 
the "biological interest rate". The properties of this solution in frameworks with a realistic demography were initially studied by Arthur and McNicoll (1978) and Lee (1980), while recent advances have been proposed by Bommier and Lee (2003) and Brito and Dilão (2010). The main difference here hinges on the fact that the demographic growth rate is endogenous. We find that there exists a monetary equilibrium which corresponds to positive aggregate assets if and only if the average age of consumers is greater that the average age of producers. It means that the average worker transfers some of her endowments to the average consumer, which is the essence of aggregate savings.

It is important to stress out that, contrary to the case with an exogenous growth rate, the monetary equilibrium is not optimal. In order to prove this, we study the optimal stationary path of our economy. The problem is similar to the one studied by Samuelson (1975) and Deardorff (1976) although we restrict ourselves to the question of characterizing the optimum and comparing it to the stationary equilibrium. The optimal stationary path is solution of the following problem:

$$
\begin{aligned}
& \max _{c(t), \tau} \int_{0}^{1} e^{-\rho t} u(c(t)) d t+\gamma v(1-\tau) \\
& \text { s.t. } \mid \begin{array}{l}
\int_{0}^{1} e^{-\nu t} c(t) d t=\int_{0}^{1} e^{-\nu t} \tilde{w}(t, \tau) d t \\
\tilde{w}(t, \tau)=\left\{\begin{array}{l}
\alpha(\tau) \text { if } t \in[\tau, \min (\tau+\theta, 1)] \\
1, \text { otherwise }
\end{array}\right. \\
\nu=\frac{\ln f(\tau)}{\tau} \\
c(t) \geq 0, \tau \in[0,1] .
\end{array}
\end{aligned}
$$

The social planner maximizes the steady state life-cycle utility subject to a static budget constraint and the demographic equation linking the population growth rate and the age at motherhood. Let us suppose that there exists an 
interior solution to Problem $(\mathrm{O})$, which is the optimum ${ }^{6}$. Then:

Proposition 2. The optimal growth rate of the population is larger than the one that prevails at the monetary equilibrium.

Proof. The resolution method for Problem (O) is the same as the one developped in Lemma 1. One may first compute the optimal consumption profile parametrized by $\tau$ and $\nu$, the latter being a function of $\tau$ defined as follows: $\nu(\tau) \equiv \ln f(\tau) / \tau$. The consumption profile, denoted $c(t, \tau, \nu(\tau))$, satisfies:

$$
c(t, \tau, \nu(\tau))=\frac{\int_{0}^{1} e^{-\nu(\tau) z} \tilde{w}(z, \tau) d z}{\int_{0}^{1} e^{-(\sigma \rho+(1-\sigma) \nu(\tau)) z} d z} e^{\sigma(\nu(\tau)-\rho) t} .
$$

The optimal age at motherhood hence solves:

$$
\max _{\tau} \int_{0}^{1} e^{-\rho t} u(c(t, \tau, \nu(\tau))) d t+\gamma v(1-\tau) .
$$

The first order condition is:

$$
\int_{0}^{1} e^{-\rho t} u^{\prime}(c(t, \tau, \nu(\tau)))\left[\frac{\partial c(t, \tau, \nu(\tau))}{\partial \tau}+\frac{\partial c(t, \tau, \nu(\tau))}{\partial \nu} \nu^{\prime}(\tau)\right] d t-\gamma v^{\prime}(1-\tau)=0 .
$$

Let us denote $\hat{\tau}$, the solution of the previous equation. It compares with the monetary equilibrium that solves

$$
\int_{0}^{1} e^{-\rho t} u^{\prime}(c(t, \tau, \nu(\tau)))\left[\frac{\partial c(t, \tau, \nu(\tau))}{\partial \tau}\right] d t-\gamma v^{\prime}(1-\tau)=0,
$$

and which will be denoted $\tau^{*}$. Provided that the problem is concave and the solution is interior, we therefore have:

$$
\hat{\tau} \geq \tau^{*} \Leftrightarrow \int_{0}^{1} e^{-\rho t} u^{\prime}\left(c\left(t, \tau^{*}, \nu\left(\tau^{*}\right)\right)\right) \frac{\partial c\left(t, \tau^{*}, \nu\left(\tau^{*}\right)\right)}{\partial \nu} \nu^{\prime}\left(\tau^{*}\right) d t \geq 0 .
$$

\footnotetext{
${ }^{6}$ Since Deardorff (1976) and Michel and Pestiau (1993), we indeed know that it is not always the case.
} 
Simple computations yield to:

$$
\hat{\tau} \geq \tau^{*} \Leftrightarrow \nu^{\prime}\left(\tau^{*}\right)\left[\bar{\alpha}_{c}-\bar{\alpha}_{y}\right] \geq 0 .
$$

Using Proposition 1, we conclude that $\hat{\tau}<\tau^{*}$, which implies that $\nu(\hat{\tau})>$ $\nu\left(\tau^{*}\right)$.

The intuition for this result is that the equilibrium does not take into account the positive impact of the population growth rate on life-cycle consumption. Indeed, a greater growth rate implies a larger production that can benefit to the elder. As a consequence, the equilibrium is not optimal.

\section{Conclusion}

Fertility choices involve three strongly interrelated decisions: the timing and spacing of births, the number of children and the quality of children. Since Becker (1960), the literature has extensively studied the tradeoff between child quality and quantity, but the first decision is much less discussed, in spite of the fact that its consequences on population growth are well documented empirically (see for instance Gustafsson, 2001). The novelty of this paper is to propose an endogenous determination of the age at motherhood. We represent this age by the age at first birth and suppose, in accordance to empirical works (Heckman and Walker, 1990), that the number of children is a decreasing function of this age. The central mechanism allowing to endogenize the age at motherhood is the comparison between the enjoyment the mother derives from spending time with her children and the opportunity cost of raising them, taking the form of a wage penalty. Recent papers endogenizing the age at motherhood have done it indirectly, considering that 
this age is a by-product of educational choices: when the mother decides to invest in her own human capital, she postpones childbearing until she has completed her education (de la Croix and Licandro, 2009).

This work could be extended in two directions. First, the analysis of the dynamics of the model could allow us to get insights on the consequences of changes in variables affecting the tempo of childbearing, as economic policies aimed at reducing the opportunity cost of raising children. Following d'Albis and Augeraud-Véron (2008), it is possible to conjecture that the economy would experience long run fluctuations. Secondly, the model could be enriched by introducing the mother's educational choices as in de la Croix and Licandro (2009), and an uncertain lifetime, which would allow us to analyze the interplay between fertility choices and mortality. The methodology developed here could then help to better understand the demographic transition 


\section{References}

[1] H. d'Albis, Demographic structure and capital accumulation. J. Econ. Theory 132 (2007) 411-434.

[2] H. d'Albis and E. Augeraud-Véron, Balanced cycles in an OLG model with a continuum of finitely-lived individuals. Econ. Theory 30 (2007) 181-186.

[3] H. d'Albis and E. Augeraud-Véron, Endogenous retirement and monetary cycles. Math. Pop. Stud. 15 (2008) 214-229.

[4] B. W. Arthur and G. McNicoll, Samuelson, population and intergenerational transfers. Int. Econ. Rev. 19 (1978) 241-46.

[5] G. S. Becker, An economic analysis of fertility, in NBER (ed.) Demographic and economic change in developed countries. Princeton, NJ: Princeton University Press (1960).

[6] A. Bommier and R. D. Lee, Overlapping generations models with realistic demography: statics and dynamics. J. Pop. Econ. 16 (2003), 135-160.

[7] R. Boucekkine, D. de la Croix, and O. Licandro, Vintage human capital, demographic trends and endogenous growth. J. Econ. Theory 104 (2002) 340-375.

[8] R. Boucekkine, D. de la Croix and O. Licandro, Modelling vintage structures with DDEs: Principles and applications. Math. Pop. Stud. 11 (2004) 151-179.

[9] R. Boucekkine, R. Desbordes and H. Latzer, How do epidemics induce behavioral changes? J. Econ Growth 14 (2009) 233-264.

[10] P. Brito and R. Dilão, Equilibrium price dynamics in an overlappinggenerations exchange economy. J. Math. Econ. 46 (2010) 343-355.

[11] A.V. Deardorff, The optimum growth rate for population: comment. Int. Econ. Review, 17 (1976) 510-515.

[12] D. de la Croix and O. Licandro, 'The Child is Father of the Man:' Implications for the Demographic Transition. Working Paper (2009).

[13] S. Gustafsson, Optimal age at motherhood. Theoretical and empirical considerations on postponement of maternity in Europe. J. Pop. Econ. 14 (2001) 225-247.

[14] J. J. Heckman and J. R. Walker, The relationship between wage and income and the timing and spacing of births: evidence from Swedish longitudinal data. Econometrica 58 (1990) 1411-1441. 
[15] Human Fertility Database. Max Planck Institute for Demographic Research (Germany) and Vienna Institute of Demography (Austria). Available at www.humanfertility.org (data downloaded on May 29, 2010).

[16] S. Kalemli-Ozcan, A stochastic model of mortality, fertility, and human capital investment. J. Dev. Econ. 70 (2003) 103-118.

[17] R. D. Lee, Age structure, intergenerational transfers and economic growth: An overview. Revue Economique 31 (1980), 1129-1156.

[18] A. J. Lotka, Relation between birth rate and death rate. Science 26 (1907) 21-22.

[19] A. J. Lotka, The stability of the normal age distribution. Proceedings of the National Academy of Sciences 8 (1922) 338-345.

[20] P. Michel and P. Pestiau, Population growth and optimality: when does serendipity hold? J. Pop. Econ. 6 (1993), 353-362.

[21] P. A. Samuelson, An exact consumption-loan model of interest with or without the social contrivance of money. J. Pol. Econ. 66 (1958), 467482.

[22] P. A. Samuelson, The optimum growth rate for population. Int. Econ. Review 16 (1975) 531-538.

[23] M. E. Yaari, Uncertain lifetime, life insurance, and the theory of the consumer. Rev. Econ. Stud. 32 (1965), 137-160. 